\title{
Diacronie
}

Studi di Storia Contemporanea

$N^{\circ} 12,4 \mid 2012$

Sulle tracce delle idee

\section{Le piazze dei mercati a Rio de Janeiro (1840-1908): tra sociabilità e modernità}

Vitor Leandro de Souza

Traduttore: Jacopo Bassi

(2) OpenEdition

Journals

\section{Edizione digitale}

URL: http://journals.openedition.org/diacronie/2479

DOI: 10.4000/diacronie.2479

ISSN: 2038-0925

\section{Editore}

Association culturelle Diacronie

\section{Notizia bibliografica digitale}

Vitor Leandro de Souza, « Le piazze dei mercati a Rio de Janeiro (1840-1908): tra sociabilità e

modernità », Diacronie [Online], № 12, 4 | 2012, documento 3, Messo online il 29 décembre 2012

consultato il 12 juin 2020. URL : http://journals.openedition.org/diacronie/2479 ; DOI : https://doi.org/ 10.4000/diacronie.2479 


\section{Diacronie}

\section{Le piazze dei mercati a Rio de Janeiro}

\section{(1840-1908): tra sociabilità e modernità*}

Vitor Leandro de SOUZA*

traduzione di Jacopo BASSI

Questo articolo intende analizzare lo sviluppo del processo di riordino del commercio di generi di prima necessità nella città di Rio de Janeiro tra la seconda metà del XIX e l'inizio del XX secolo attraverso la costruzione e la regolamentazione delle piazze dei mercati. Si cercherà, attraverso testimonianze rinvenibili nella stampa, nella letteratura, nella documentazione amministrativa e nelle immagini, di ricostruire come questi cambiamenti furono vissuti dai frequentatori dei mercati e come furono sfruttati dall'amministrazione pubblica per realizzare quelle infrastrutture conformi all'ideologia modernizzatrice dell' inizio del XX secolo.

\section{Rio, città-problema}

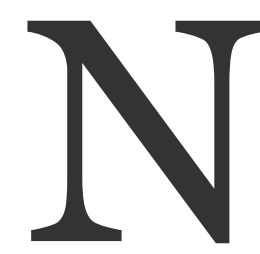

egli ultimi decenni del XIX secolo la città di Rio de Janeiro era la più grande dell'Impero e, in quanto tale, sentiva e viveva intensamente i cambiamenti politici, economici e sociali avvenuti nella seconda metà dell'Ottocento. I censimenti rivelano un'accelerazione nell'incremento della popolazione «intimamente legato alla emigrazione degli schiavi liberati dalle zone rurali verso quella urbana, allintensificazione dell'immigrazione e ai miglioramenti nelle condizioni di vita apportate dalla bonifica [delle terre]»1.

\footnotetext{
* Questo articolo è una versione rivista e ampliata di una parte della tesi del Curso de Graduação in Storia, intitolata "Ordem na praça: ação Municipal nas Praças de Mercado do Rio de Janeiro nos séculos XIX e XX”, presentata nel luglio 2011 all'Universidade Federal Fluminense, sotto la direzione della Professoressa Laura Antunes Maciel.
} 
Oltre ad essere il principale centro di consumo, la capitale era il centro del potere politico, decisionale e d'affari - evidente dall'intenso traffico del suo porto - e dello smercio della maggior parte della produzione caffearia destinata all'esportazione ${ }^{2}$.

La città-problema, come viene definita da Maria Yedda Linhares ${ }^{3}$, presentava difficoltà nel predisporre alloggi dignitosi per la sua crescente popolazione, oltre a gravi problemi infrastrutturali e nell'approvvigionamento di generi alimentari. Nell'area urbana erano localizzate le opportunità di lavoro, di commercio, i servizi e le abitazioni (prevalentemente abitazioni collettive), il che rendeva le freguesias ${ }^{4}$ centrali, aree contrassegnate da un'elevata concentrazione di famiglie per edificio.

Seguendo una consuetudine portoghese 5 , fu nell'area portuale - attualmente conosciuta come Praça 15 - che, a partire dalla metà del XVII secolo, furono introdotte fiere e mercati, creando così un punto di commercio tradizionale della città di Rio de Janeiro. Inizialmente il funzionamento era caratterizzato dalla disorganizzazione, in baracche senza un "allineamento o una simmetria definite» 6 ; un intenso e colorato negoziare si svolgeva tra i banchi del pesce, come in una «fiera permanente»7, o per mezzo dei bottegai, delle negras de tabuleiro ${ }^{8}$ e degli ambulanti, che rifornivano la città nelle vie, nei vicoli e nelle piazze pubbliche.

${ }^{1}$ CHALHOUB, Sidney, Trabalho, Lar e Botequim: o cotidiano de trabalhadores no Rio de Janeiro da belle époque, Campinas, Editora da Unicamp, 1986, p. 24.

2 Per un panorama della situazione affrontata da Rio de Janeiro in questo periodo si veda: DAMAZIO, Sylvia F., Retrato social do Rio de Janeiro na virada do século, Rio de Janeiro, EdUERJ, 1996.

3 LINHARES, Maria Yedda, História do Abastecimento: uma problemática em questão (15301918), Brasília, Binagri, 1979.

4 La parola orginariamente indicava una sottocircoscrizione ecclesiale della diocesi; il termine è poi passato a indicare una circoscrizione amministrativa. Sulla formazione e lo sviluppo delle freguesias a Rio de Janeiro si veda: FRIDMAN, Fania, «Freguesias fluminenses ao final do Setecentos», in Revista do Instituto de Estudos Brasileiros, 49, 2009, pp. 91-143,

URL: < http://www.revistasusp.sibi.usp.br/scielo.php?script=sci_arttext\&pid=Soo20$38742009000200005 \& \operatorname{lng}=$ pt\&nrm $=$ iso $>$ [consultato il 27 settembre 2012] [n.d.t].

5 MOTT, Luiz, «Feiras e mercados: pistas para pesquisa de campo», in FERRETTI, Sergio (a cura di), Reeducando o olhar: estudos sobre feiras e mercados, São Luís, Edições UFMA PROIN, 2000, pp. 13-34.

${ }^{6}$ COSTA, Nelson, Rio de Ontem e de Hoje, Rio de Janeiro, Leo, 1958, p. 211.

7 FARIAS, Juliana Barreto, «Mercado em greve: Protesto e organização dos trabalhadores do pequeno comércio no Rio de Janeiro - Outubro, 1885», in Anais da Biblioteca Nacional, 127, 2010, p. 4.

${ }^{8}$ Le Negras de tabuleiro erano venditrici ambulanti, schiave e affrancate, espressione di una certa specializzazione della mano d'opera generata dall'aumento di popolazione nei centri urbani e dallo sviluppo di nuove attività economiche. Sul tema si veda: REIS, Liana Maria, «Mulheres de ouro: as negras de tabuleiro nas Minas Gerais do século XVIII», in Revista do Departamento de História, 8, 1/1989, pp. 72-85; DIAS, Maria Odila Leite da Silva, «Nas Fímbrias da Escravidão Urbana: Negras de. Tabuleiro e de Ganho», in Estudos Econômicos, 15, 1985, pp. 89-109 [n.d.t.]. 


\section{La piazza del Mercado da Candelária}

A dispetto delle azioni intraprese precedentemente dal potere pubblico, solamente nel 1834 si diede inizio alla costruzione di un locale specifico destinato al commercio di generi di consumo. Il Mercado da Candelária rappresentava un tentativo di ordinamento del commercio delle freguesias centrali della città da parte dell'amministrazione comunale. Successivamente alla pubblicazione di un bando per la costruzione, dal momento che non si trovarono soggetti interessati, la stessa amministrazione comunale si impegnò a realizzare l'opera.

Il progetto architettonico affidato al francese Grandjean de Montigny, «comprendeva un vasto edificio con due piani, di pianta rettangolare, con arcate sopra un patio centrale [...], i negozi erano accessibili esclusivamente dall'interno del mercato»9. Sprovvisto di fondi, il Consiglio comunale decise di non eseguire il progetto originale, adattandolo (figura 1) e costruendo solamente un piano.

Nel 1844 fu fissato un regolamento ${ }^{10}$ per il funzionamento del Mercado da Candelária, che stabiliva che sarebbero state a carico della Prefettura l'amministrazione e la vigilanza del mercato, la determinazione della quantità di bancarelle, la frequenza e la modalità di pagamento delle stesse, così come la regolazione degli affitti degli spazi all’interno dell'edifico. Nel 1869 la piazza del Mercado da Candelária venne concessa all'amministrazione privata «per nove anni, dall'Illustrissimo Consiglio comunale, a Aureliano Dias da Costa Cabral e al tenente-colonello Antônio José da Silva [il] quale in conseguenza di ciò organizzò un'impresa per realizzare i miglioramenti [menzionati] dal contratto ${ }^{11}$. L'edificio fu ampliato, recependo quelle migliorie menzionate nell'Almanak Laemmert (un piano superiore e opere di copertura dello stabile); in questo periodo questi interventi costavano caro a quelli che prendevano in affitto una bancarella al Mercado da Candelária ${ }^{12}$.

\footnotetext{
9 FRIDMAN, Sergio A., GORBERG, Samuel, Mercados no Rio de Janeiro. 1834-1962, Rio de Janeiro, S. Gorberg, 2003, p. 12.

${ }^{10}$ Il regolamento è riportato in FRIDMAN, Sergio A., GORBERG, Samuel, op. cit., pp. 12-50.

11 ALMANAK LAEMMERT, Almanak Administrativo Mercantil e Industrial do Império do Brazil para 1844, Rio de Janeiro, Laemmert \& C, 1844, p. 839.

${ }^{12}$ Sul passaggio dal libero commercio alla regolamentazione e all'affitto dei locali per scopo di lucro si veda MACIEL, Laura Antunes, De SOUZA, Vitor Leandro, «Ordem na praça: normas e exercício de administraçao em mercados do Rio de Janeiro», in Passagens, 4, 1/2012,

URL: < http://www.historia.uff.br/revistapassagens/artigos/v4n1a32012.pdf > [consultato il 7 novembre 2012] [n.d.t.].
} 


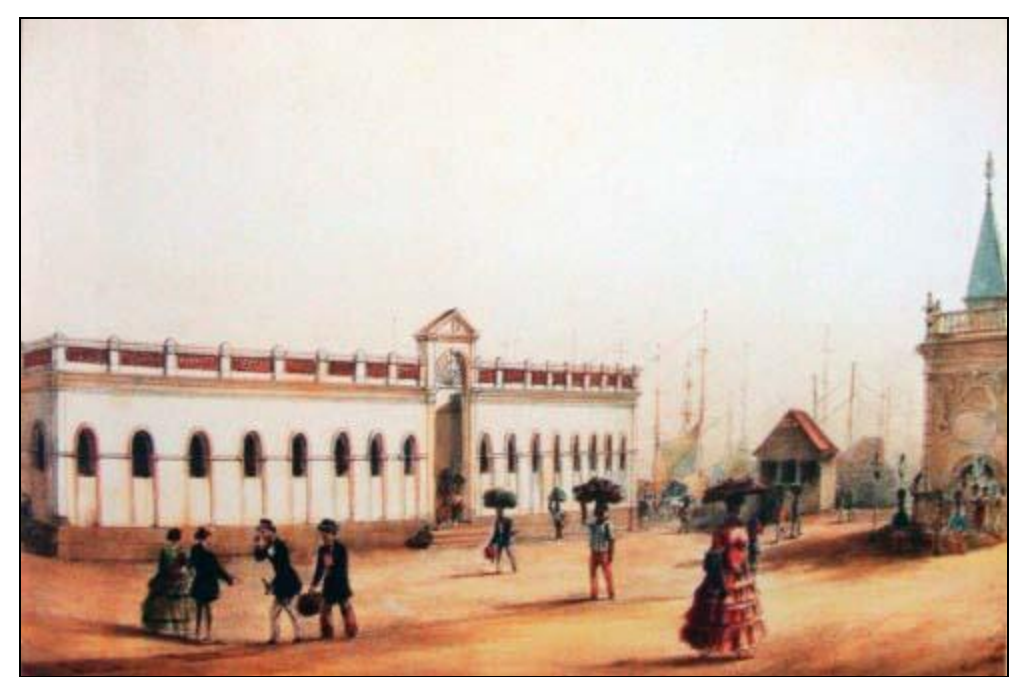

Fig. 1 - BERTICHEN, Pieter Godfred, Largo do Paço, Mercado da Candelária 1840-186o.

(Litografia, cor). Collezione del Museo Imperiale - Petrópolis/RJ.

Altre piazze del mercato erano in funzione in città nello stesso periodo ed erano amministrate in modo simile. Nel quartiere di Gamboa funzionava, sin dal 1857, il Mercado da Harmonia, ceduto al generale Lazaro José Gonçalves Junior, per l'utilizzo e l'usufrutto per l'arco di vent'anni. Eppure lo scarso movimento e la crisi delle abitazioni che sconvolgeva la città fece sì che una parte dell'edificio fosse utilizzato come alloggio collettivo, giungendo a essere definito come «un cortile immondo [nel quale] tutto si vendeva, tranne i generi di prima necessità»13, imputato di provocare numerosi problemi igienici, incluso un caso di peste bubbonica.

Nella freguesia di Glória fu costruito, tra il 1856 e il 1858, un mercato in sostituzione delle baracche improvvisate che erano utilizzate per il commercio nei pressi del litorale. L’incarico per l'esecuzione dell'opera era stato affidato a Inácio de Barros Vieira Cajueiro, il cui contratto con il Consiglio comunale prevedeva la conclusione dell'opera entro due anni: come contropartita la municipalità offriva «per enfiteusi perpetua i terrenos de marinha ${ }^{14}$ esistenti nel luogo»15 e pieni poteri per l'espropriazione e la demolizione degli immobili che avessero impedito l'esecuzione delle opere. L’edificio del Mercato de Praça da Glória, che aveva lo stesso utilizzo del Mercado de Praça da Harmonia, fu gradualmente occupato dalle famiglie povere, che

\footnotetext{
13 FRIDMAN, Sergio A., GORBERG, Samuel, op. cit., p. 38.

${ }_{14}$ Conformemente al Decreto-Legge $9760 / 46$ sono considerati terrenos de marinha quelli che occupano la fascia di terreno litoraneo fino a 33 metri di distanza dalla linea delle aree inondate dalla mareggiata dell'anno 1831.

URL:

<http://www.aracaju.se.gov.br/userfiles/emurb/licenciamento_de_Obras/leis_federais_estad uais/dec_lei_9760_46.pdf $>$ [consultato il 27 settembre 2012] [n.d.t.].

15 FRIDMAN, Sergio A., GORBERG, Samuel, op. cit., pp. 42-46.
} 
avevano necessità di abitare vicino a luoghi in cui potevano generarsi opportunità di lavoro e che si trovavano ad essere abbandonate dal potere pubblico.

Era inoltre meta di «persone disoccupate che durante la notte vi producevano ogni sorta di sozzura e di assembramento» ${ }^{16}$, il che scatenò le proteste degli abitanti attigui all'edificio: il Mercado da Glória rimase in rovina fino al 1903 quando fu demolito per fare spazio alle opere di costruzione dell'Avenida Beira Mar. La "civilizzazione" raggiungeva anche questo punto della città, sostituendo il Mercado da Glória con una bella piazza, dotata di una fontana per abbellire la strada che univa le freguesias centrali alle zone dell'asse sud della città, roccaforte delle classi aristocratiche.

\section{Gli usi del Mercado da Candelária}

La piazza del Mercado da Candelária si consolidava come un importante spazio dove si sviluppavano differenti sociabilità, che richiamavano l'attenzione dei cronisti dell'epoca:

C'è una grande concorrenza e movimento di popolazione non solo nel recinto del mercato, ma anche nella Praça das Marinhas. E la mescolanza di differenti classi di popolazione, il disordine che sembra esserci tra venditori e compratori, l'assembramento di uomini, donne, bambini e schiavi che o si svagano o si recano in cerca d'ogni sorta di prodotti che qui si incontrano, è uno spettacolo curioso e caratteristico delle consuetudini del paese ${ }^{17}$.

Il "disordine" narrato dal cronista ci aiuta a comprendere l'intenso commercio, le attività svolte negli organi legati all'Amministrazione dello Stato, così come quelle nell'imbarco e sbarco dei passeggeri. Era un luogo di intenso traffico tra la Città Vecchia e la Città Nuova e, al contempo, tra le periferie, soprattutto a seguito della diffusione dei battelli a vapore - avvenuta negli anni Quaranta dell'Ottocento - che permise il collegamento tra Saco do Alferes e Botafogo e tra São Cristovão e la spiaggia Dom Manuel, con una fermata nell'insenatura del Valongo. Importanti opere infrastrutturali quali terrapieni, rampe e scale rendevano possibile il "miscuglio" che infastidiva Azevedo ${ }^{18}$ nella sua memoria.

\footnotetext{
16 Ibidem.

17AZEVEDO, Moreira de, Rio de Janeiro: sua história, monumentos, homens notáveis, usos e curiosidades, vol. 2, Rio de Janeiro, B. Garnier, 1877, pp. 322-323.

18 Per maggiori dettagli sugli interventi nell'area portuale della città si veda: LAMARÃO, Sérgio Tadeu de Niemeyer, Dos trapiches ao porto: um estudo sobre a área portuária do Rio de
} 
Il traffico di lavoratori, venditori, escravos de ganho ${ }^{19}$, viaggiatori, politici, maggiorenti che si recavano a corte per trattare affari o cercare appoggio politico, dava corso al curioso spettacolo e dimostrava come questa zona non fosse solamente un luogo di semplici relazioni commerciali, ma anche di incontri, rapporti e intensi interscambi sociali. In questo senso, la determinazione di un luogo designato a centralizzare la vendita dei prodotti risulta importante ai fini del consolidamento di queste relazioni tra le diverse classi sociali. Non che fosse stata abbandonata la pratica della vendita per strada, ma nella dinamica che si veniva realizzando nella piazza del mercato il consumatore trovava nello stesso luogo una maggior varietà di prodotti.

Il mercato funzionava come un emporio, con la funzione di locale di incontro tra facchini, spazzini, barcaioli, domestiche, venditori ambulanti, rendendo possibile il rafforzamento dei legami e sviluppando le opportunità di socializzazione. I vendedores de cestos, per esempio, portavano - oltre che prodotti - anche notizie, quasi "contaminando" la città: diffondendo tra le soglie delle case e gli stretti vicoli le novità raccolte nei corridoi del mercato, nelle botteghe, nelle macellerie e nelle trattorie, riportavano dal mercato gli episodi ascoltati e visti nelle strade delle freguesias più interne.

O ancora, possiamo supporre che la negoziazione tra i venditori e domestiche che si recavano a far compere per conto dei loro padroni, potesse indicare le difficoltà finanziarie delle famiglie - quelle considerate come le più "tradizionali" o quelle che occupavano una posizione sociale riconosciuta -, evento non improbabile a fronte del rincaro dei prezzi. È ipotizzabile che i più intimi segreti della casa dei padroni fossero resi pubblici lì, tra le bancarelle del pesce e degli ortaggi, condivisi con i venditori o, allo stesso modo, con altre domestiche ${ }^{20}$.

Il traffico intenso - all'interno e all'esterno - del mercato iniziava presto: «Già prima delle sei è una babele rumorosa, dove un mondo si muove, schiamazza e si espande ${ }^{21}$. I protagonisti di queste relazioni, oltre ai venditori e agli acquirenti, o a

Janeiro, Rio de Janeiro, Secretaria Municipal de Cultura, Turismo e Esportes, Departamento Geral de Documentação e Informação Cultural, Divisão de Editoração, 1991, pp. 40-44.

${ }_{19}$ L'escravo de ganho lavorava fuori dalla casa del padrone. Vendeva nei mercati ogni sorta di mercanzia - talvolta negoziava con i clienti per conto delle prostitute - di norma senza essere sotto il diretto controllo del padrone; era però tenuto a versargli una cifra fissa, solitamente con cadenza settimanale, tenendo per sé il necessario per il proprio sostentamento. s.v. «Escravo de ganho», in MOURA, Clóvis (a cura di), Dicionário da escravidão negra no Brasil, São Paulo, Editora da Universidade de São Paulo, 2004, p. 150 [n.d.t.].

${ }^{20}$ L'ipotesi è stata suggerita a partire dalle relazioni tra domestiche e padroni esposte da LAUDERDALE GRAHAM, Sandra, House and street: the domestic world of servants and masters in Nineteenth-Century Rio de Janeiro, Austin, University of Texas Press, 1992.

${ }^{21}$ EDMUNDO, Luís, O Rio de Janeiro de meu tempo, Rio de Janeiro, Editora Conquista, 1957, p. 60. 
quei frequentatori che in un giorno normale potevano essere impiegati in quei corridoi affollati di gente, erano escravos de ganho, facchini, immigrati poveri in cerca di un posto di lavoro, domestiche, liberti, prostitute ${ }^{22}$ e accattoni delle colline della zona centrale. Lì potevano trovare una quantità di opportunità e occasioni per sopravvivere ad una realtà escludente che si consolidava con i mutamenti socioeconomici della seconda metà del secolo.

Attraverso le cronache e le immagini possiamo venire a conoscenza degli usi "altri" del mercato, frequentemente battuto da ispettori fiscali e poliziotti, nell'intento - ogni volta più deciso - di imporre l'ordine. La chiarezza di João do Rio ci permette di comprendere il dissolvimento della concezione classica del mercato inteso solamente come luogo di acquisto e vendita di prodotti; nella sua cronaca talvolta il mercato si trasforma in un angolo "disgiunto dalla città":

Nella piazza si trovava l'abbondanza, la ricchezza, la miseria e il vagabondaggio. A fianco dei ragazzotti che lavoravano sodo fin dall'alba tra montagne di vegetali e cumuli sanguinanti di carne, inseguendo fortune fatte con le braccia nel disimballare cipolle e aglio, vivevano e morivano affamati monelli squallidi, vagabondi forestieri, tutta la mota del crimine, dell'orrore della prostituzione $[\ldots]^{23}$.

Pur apparendo chiaro il suo preconcetto, il cronista ci permette di identificare le prostitute che tra tranci di merluzzo e sacchi di farina offrivano il loro corpo a pagamento. Al cadere della notte avveniva ogni sorta di promiscuità tra "montagne di ortaggi" e "carni sanguinanti” come, per esempio, il consumo di oppio. L'autore unisce la povertà e la ricchezza; nello stesso spazio si registra la presenza di ladri, zingari, mendicanti e lavoratori poveri che non riuscivano più a lavorare a causa di qualche tipo di problema di salute.

\footnotetext{
${ }^{22}$ Sulla questione della prostituzione e della sessualità a Rio de Janeiro si veda: FIGARI, Carlos, As outras cariocas. Interpolações e identidades homoeróticas no Rio de Janeiro. Século XVII a $X X$, Belo Horizonte-Rio de Janeiro, Editora UFMG-Editora da IUPERJ, 2007, pp. 108-110.

${ }_{23}$ Paulo Barreto, sotto lo pseudonimo di João do Rio, fu un importante cronista della fine del secolo. La cronaca intitolata $O$ Velho Mercado fu pubblicata nella «Gazeta de Notícias», il 12 gennaio 1908, p. 1. Successivamente fu incluso nel libro di cronache: RIO, João do [BARRETO, Paulo], Cinematographo: crônicas cariocas, Porto, Lello \& Irmão, 1909. Sulla visione della città di João do Rio QUELHAS, Iza, «A Cidade nas Crônicas de João do Rio», in Cadernos do Tempo Presente, 7, 2012,

URL: < http://www.getempo.org/revistaget.asp?id_edicao=33\&id_materia=129 > [consultato il 3 novembre 2012].
} 
In mezzo alla «Babele rumorosa» ${ }^{24}$, nella bellezza caotica del «cielo, molto azzurro» e del sole «che spande tutta la sua luce dorata», tra battelli, vele bianche, isole, fortificazioni e vie animate che sboccavano sulla banchina, «la vita tumultuosa della città vibrava in un rumore di apoteosi ed era ancor più intensa, più brutale, più strillata, in quello spazio del mercato, in quella parte della rampa, viscida di immondizie e di vizi» 25 .

Nelle sue descrizioni, il flâneur cercava di svelare il lato più oscuro dello "stomaco della città”, prassi che era quasi una legge tra i suoi colleghi giornalisti francesi: quella di guidare la "gente raffinata" con sicurezza attraverso le aree piene di «immondizie e di vizi» della capitale federale, rivelando pratiche e stili di vita che la società fingeva di ignorare. Con le sue parole l'autore persegue l'obiettivo di condurre i suoi lettori all'eccitante e tragica visione «che la miseria tesse nell'ombra della notte attraverso questa misteriosa città» ${ }^{26}$.

Nell'intento di offrire ai suoi lettori un maggior realismo, João do Rio talvolta riproduce la voce delle persone che incontra nelle sue avventure per la città. Un buon esempio ci viene fornito quando ci riferisce delle rivendicazioni degli scaricatori ${ }^{27}$, quasi sempre «gli stranieri poveri», obbligati a cercare il sostentamento così - per molti, del resto, era l'unica opportunità a disposizione - lavorando molto, e sotto al sole, per una retribuzione bassissima.

Secondo il cronista uno scaricatore, cosciente della situazione, affermò: «I signori non sanno che questo paese è ricco, ma che si muore di fame? [...] Il capitale è nelle mani di un gruppo ristretto e la gente rimane sempre più del tutto senza lavoro». E continuava: «Ai padroni non interessa sapere se restiamo inattivi per eccesso di manodopera». Per quel lavoratore era facile incontrare anche al mercato molti "lavoratori inattivi" che, per le condizioni di indigenza a cui erano sottoposti, molte volte avevano come unico sostentamento gli avanzi di cibo.

\footnotetext{
24 Nell'opera più famosa di João do Rio, intitolata A Alma encantadora das ruas, furono riuniti i testi publicati tra 1904 e 1907 nella «Gazeta de Notícias» e nella rivista illustrata «Kosmos»; questi periodici circolavano principalmente tra uomini e donne dell'élite, classi medie (che aspiravano all'ascesa sociale), alcuni studenti e scrittori. Le citazioni contenute nei paragrafi successivi sono presenti nel testo di RIO, João do [BARRETO, Paulo], O que se vê nas ruas. Pequenas profissões, in RIO, João do [BARRETO, Paulo], A Alma encantadora das ruas, Rio de Janeiro: Organizações Simões, 1951. Disponibile all'URL:

< http://www.dominiopublico.gov.br/download/texto/bnooo039.pdf > [consultato il 19 ottobre 2012].

25 Ibidem.

${ }^{26}$ Ibidem.

${ }_{27}^{27}$ RIO, João do [BARRETO, Paulo], Os Trabalhadores de Estiva, in RIO, João do [BARRETO,

Paulo], A Alma encantadora das ruas, cit.
} 
Il reporter del "sottomondo carioca" ci aiuta a soffermarci maggiormente, nella frenesia della città, su un personaggio: «José Vizuvi, figlio di un immigrato italiano, il famoso mendicante Vizuvi». Di buon'ora, «alle 5 di mattina, esce alla ricerca delle pagnotte che i panettieri sono soliti lasciare alle finestre e alle porte di alcune case». Con la condotta malandrina ${ }^{28}$ di un ladro, comprendendo che «la finestra è alta, si serve di un bastone munito di gorbia». Avendo appreso la tecnica di sopravvivenza dal padre, trascorreva la parte rimanente del «giorno nel mercato e nei quartieri centrali» per compiere il suo "lavoro". Il cronista narra il profilo di un altro "mariuolo", di nome João da Silva, abitante del centro, di 13 anni di età, nero, che è dedito a «lavori poco puliti». La madre di João da Silva, disoccupata, finge di essere cieca per guadagnarsi da vivere mendicando nel Largo do Machado; fuggita con l'amante, che le ruba tutto il denaro, probabilmente il figlio assiste al suo maltrattamento dando vita alla «tragedia strappalacrime» evocata dall'autore ${ }^{29}$. Dunque, possiamo capire che la città presentata da João do Rio non è solamente quella dei caffè e delle boutique della Rua do Ouvidor, e ancor meno delle pasticcerie e delle passeggiate nella Avenida Central.

Il termine "classi pericolose" è illustrato dallo storico Sidney Chalhoub30 come un concetto adottato dall'élite politica brasiliana in associazione con l'idea di povertà. Stando così le cose, bastava essere poveri per rappresentare un pericolo. È doveroso ricordare anche che, soprattutto nella città di Rio de Janeiro, a causa della forte presenza di neri - affrancati dopo il $1888^{31}$ - e di immigrati europei poveri che si trovavano ad essere socialmente esclusi, privi di un lavoro degno e costretti ad abitare nei cortili e nelle abitazioni collettive delle zone centrali della città, questi erano visti non solamente come una minaccia alla proprietà, ma anche alla salute. Come ci spiega l'autore «i cortili sono visti tanto come un problema per il controllo sociale dei poveri

${ }^{28}$ L'autore usa il termine malandragem, che fa riferimento a un vasto campo di significati. Il malandro, colui che vive di espedienti, di truffe e di piccoli furti, infedele alle donne ed edonista è divenuto una figura popolare brasiliana, prototipo dell'antieroe; furbo e astuto riesce a carpire la fiducia del prossimo e a volgere le situazioni a proprio vantaggio. Sul tema si vedano: CANDIDO, Antonio, Dialectica da Malandragem, in CANDIDO, Antonio, O Discurso e a cidade, São Paulo, Livraria duas cidades, 1970, pp. 19-55.; DaMATTA, Roberto, Carnavais, malandros e heróis. Para uma sociologia do dilema brasileiro, Rio de Janeiro, Zahar Editores, 1979 [n.d.t.].

29 RIO, João do [BARRETO, Paulo], Os que começam in RIO, João do [BARRETO, Paulo], $A$ Alma encantadora das ruas, cit.

$3^{\circ}$ CHALHOUB, Sidney, Cidade febril: cortiços e epidemias na Corte Imperial, São Paulo, Companhia das Letras, 1996. pp. 20-31.

${ }^{31}$ Nel 1888 la Lei Áurea abolì definitivamente la schiavitù in Brasile; la legge rappresentò l'approdo di un processo legislativo che mirava a una graduale abolizione della servitù. Sílvia Hunold Lara e Sidney Chalhoub hanno sostenuto il ruolo attivo giocato dagli schiavi per ottenere l'affrancamento. Si vedano CHALHOUB, Sidney, Cidade febril cit.; LARA, Silvia Hunold, Fragmentos setecentistas: escravidão, cultura e poder na América portuguesa, São Paulo, Companhia das Letras, 2007 [n.d.t.]. 
quanto come una minaccia alle condizioni igieniche della città»32. La preoccupazione era che quei cortili si trasformassero in spazi interni alla città dove lo Stato non aveva un controllo effettivo, visti come fonti di vizi, promiscuità e trasgressioni della morale e dell'ordine che si desiderava imporre.

Seppure di fronte al baratro socioeconomico che si apriva tra le classi, in qualche modo questi stili di vita, che apparentemente abitavano mondi tanto distanti, si incrociavano: si trovavano al mercato, in chiesa, nelle case di tolleranza o sul Bonde. Sebbene l'idea fosse quella di escludere e allontanare sempre più le "classi pericolose" dal centro cosmopolita della capitale federale, in alcune occasioni - o luoghi, come il mercato - queste vite si incontravano.

Questo scontro tra gli usi diversi della città può esser percepito tra le bancarelle del mercato, al molo e nella diversità delle persone che lì si riunivano. In definitiva, ricchi e poveri, domestiche e padroni, avevano bisogno di nutrirsi; benché per alcuni le difficoltà nell'acquistare generi alimentari fossero maggiori, per quei poveri che vivevano accalcati nei cortili delle freguesias centrali si trattava comunque di un luogo di opportunità.

Lo scrittore Luis Edmundo ci aiuta a ricostruire come si rapportava la città dei ricchi - moderna e cosmopolita - con quella dei poveri - coloniale, arretrata, nera dove «dopo una buona nottata [in alcuni café o a teatro nello stile dei cabarè francesi], l'elegante si trova per mangiare in questi antri, di mattina, ostriche accompagnate dal vino bianco»33. Nella narrazione di Edmundo, «elegante» e «antro» si incontrano, palesando bene l'opinione sul luogo. La «Praia do Peixe, rumorosa, ciarliera, si manifesta, da lontano, per l'enorme gazzarra che si leva e per il cattivo odore che esala. È un mercato degno di una città coloniale»34. La modernità che alcuni cercavano di imporre sin dalla fine del XIX secolo si incontrava con la società "coloniale".

La rivista «Fon-Fon» approva quella che sembra essere una pratica frequente nella società carioca, ancorché lasci emergere il preconcetto di alcuni su queste abitudini: «Mlle Sans Titre, Tijuca. Questa storia di mangiare ostriche all'alba nella piazza del mercato in compagnia di due interessanti signorine può esserle spiegata solamente dallo stesso Figueiredo Pimentel»35. Suggerendo che la spiegazione avrebbe potuto essere fornita da Figueiredo Pimentel, l'ironia del redattore della rivista «Fon-Fon» è indirizzata contro l'autore della massima O Rio civiliza-se!; nell'intento del redattore

32 CHALHOUB, Sidney, Trabalho, Lar e Botequim: o cotidiano dos trabalhadores no Rio de Janeiro da belle époque, Campinas, Editora da Unicamp, 2001, p. 31.

33 EDMUNDO, Luís, O Rio de Janeiro de meu tempo, cit., pp. 59-60.

34 Ibidem

35 «Caixa de Gazolina», in Fon-Fon!, 14, 18 janeiro 1908. 
solamente il cronista della modernità avrebbe potuto spiegare le abitudini primitive che convivono nella grande metropoli.

\section{Il Novo Mercado e il sogno tangibile dell'Europa}

Attenti a tutto ciò che accadeva in Europa, gli intellettuali e le autorità politiche brasiliane desideravano - a qualunque costo - eliminare qualsiasi residuo del fatto che il Brasile fosse stato una colonia portoghese e una nazione con una forte presenza di schiavi. Era pertanto necessario modificare in profondità gli spazi della città e le abitudini degli abitanti affinché corrispondessero all'ideale di civilizzazione. Nella realtà, ripensare un nuovo modo di commercializzare gli alimenti nella capitale della Repubblica fu solamente una delle iniziative intraprese dagli organi di governo municipale e federale per elevare la città al livello delle grandi capitali europee. Opere di "civilizzazione" nel centro urbano della città di Rio de Janeiro, come l'apertura dei grandi viali, profondi interventi di rimodellamento nel porto, il "bota abaixo" 36 della fine del secolo, colpivano principalmente gli strati più poveri della società che trovavano nella zona centrale della città l'unica opzione percorribile di accesso ai posti di lavoro, di alloggio e sostentamento.

Tutto questo lusso [delle costruzioni nella zona centrale della città] e splendore è visibile dall'alto delle colline della Favela, da quelli che, anni prima, risiedevano nei cortili, nel cuore della città. Rio si modernizza, benché la maggior parte dei suoi abitanti non sappia bene il significato di questa parola37.

Per la modernizzazione del Mercado da Candelária l'opzione prescelta fu costruire una nuova piazza del mercato. Le discussioni e i negoziati per la costruzione di una nuova piazza presero avvio dalla metà degli anni Ottanta dell'Ottocento, ma fu sotto l'impulso delle azioni messe in opera durante la gestione del prefetto Francisco Pereira Passos che un nuovo mercato municipale, situato nei dintorni di Praia D. Manoel, cessò di essere semplicemente un progetto.

\footnotetext{
36 "Bota-abaixo" è il nome popolare con cui vengono designati gli interventi di riforma sanitaria e urbanistica realizzati all'inizio del XX secolo, quando Francisco Pereira Passos ricoprì la carica di prefetto. ROCHA, Oswaldo Porto, A era das demolições: cidade do Rio de Janeiro: 18701920, Rio de Janeiro, Secretaria Municipal de Cultura, Departamento Geral de Documentação e Informação Cultural, Divisão de Editoração 1986; BENCHIMOL, Jaime Larry, Pereira Passos: um Haussmann tropical. A renovação urbana da cidade do Rio de Janeiro no início do século $X X$, Rio de Janeiro, Biblioteca carioca,1992 [n.d.t.].

37 ROCHA, Oswaldo Porto, op. cit., p. 98.
} 
L'avvio dell'opera avvenne nel 1903 e l'inaugurazione del mercato municipale della città di Rio de Janeiro - chiamato anche Mercado Central - ebbe luogo il 14 dicembre del 1907, anche se questo funzionò regolarmente solo a partire dal febbraio 1908. João do Rio testimonia le differenze di una piazza rispetto all'altra e ne registra l'affanno e le incertezze nella cronaca $O$ velho mercado ${ }^{3}$. L'autore ci aiuta a immaginare ciò che avvenne in quel giorno rannuvolato, coperto, cupo, triste. Come tanti altri giorni nuvolosi, nei quali l'allegria solare, caratteristica di questa città, dà luogo a una realtà grigia di incertezze e mutamenti, «Quel cambiamento era il maggiore di tutti, era un'operazione di chirurgia urbana, portava a modificare totalmente la Rio di una volta, la mobilitazione dello stomaco stesso dalla città verso un altro luogo»39.

Il cronista, scrivendo sul vecchio mercato, ci aiuta a comprendere il sentimento di quelli che in diversi modi frequentavano il Mercado da Candelária e di come costoro si sentissero afflitti dal modo in cui i cambiamenti annunciati avrebbero potuto intaccare la loro vita quotidiana, dal momento che, a suo parere:

Il progresso, l'igiene, ciò che è comodo, livella anime, gusti, abitudini; la civilizzazione è l'uguaglianza in alcuni capisaldi, che di comune accordo sono ritenuti degni di ammirazione; così, come le donne occidentali usano gli stessi cappelli, le stesse stoffe, la stessa andatura; così, come due uomini ben vestiti devono fatalmente avere la stessa forma del bavero della giacca e del cappello, tutte le città moderne hanno grandi viali, piazze squadrate, mercati e palazzi di ferro, vetro e ceramica ${ }^{40}$.

Infine Rio, sforzandosi di cessare di essere una città esotica, coloniale e contrassegnata dalle «tante tradizioni» secondo la definizione di João do Rio, «finì per spogliarsi di queste con indifferenza». L'autore non cessa di riportare il suo stupore dinanzi alla rapidità di questi cambiamenti: infatti è questo tono di malinconia, unito a un certo malcontento e alla critica, che orienta tutta la narrazione di O Velho Mercado.

Per capire meglio questo cambiamento dello "stomaco della città" nella freguesia di Candelária attraverso un'altra fonte, si può osservare come due dei principali giornali, che registrarono l'inaugurazione del mercato municipale della fine del 1907, argomentassero la necessità della nuova costruzione.

${ }^{38}$ Le descrizioni che seguono sono tratte da RIO, João do [BARRETO, Paulo] in Gazeta de Notícias, 12 janeiro 1908, p. 1.

39 Ibidem.

40 Ibidem. 
Un reportage del «Jornal do Brasil»»11 del 14 dicembre fornisce, nel suo primo paragrafo, una giustificazione per la realizzazione del nuovo mercato finalizzato al «commercio dei prodotti di consumo quotidiano»: il bisogno della città è di possedere un edificio all' «altezza del progresso» auspicato per la capitale federale; si mostra poi la rapidità con cui lo sviluppo della città si andava realizzando: in definitiva, si trattava della capitale della Repubblica degli Stati Uniti del Brasile. Si faceva urgente l'esigenza della costruzione di un edificio che cancellasse definitivamente la traccia degli aspetti coloniali.

Rio de Janeiro, che in un breve lasso di tempo ha proceduto di sviluppo in sviluppo, aveva bisogno di dotarsi di un'istituzione all'altezza del suo progresso, che potesse essere destinata al commercio dei prodotti di consumo quotidiano. È prendendo in considerazione questa necessità che $[. .$.$] si inaugura oggi ufficialmente il maestoso$ edificio costruito per questa finalità in Praça D. Manuel.

Il testo prosegue descrivendo il nuovo edificio del mercato della città di Rio de Janeiro, con i suoi 500 spazi destinati all'accoglienza dei commercianti e dei differenti prodotti in una struttura di ferro (figura 2) disegnata dall'ingegnere Alfredo de Azevedo Marques con materiali importati da Bruxelles. Oltre a sottolineare la modernizzazione offerta dall'edificio ai commercianti e ai fruitori del mercato, il «Jornal do Brasil» metteva in risalto i materiali utilizzati nella costruzione, interamente in ferro, pietra da taglio e piastrelle, suggerendo come non fosse stato impiegato «alcun materiale soggetto a incendio».

L’imponente edificio era il più grande costruito in Brasile utilizzando ferro, ispirato ai mercati europei, in particolar modo a quello parigino di Les Halles. Come ebbe occasione di sostenere José Vanildo Júnior:

Simbolo della tanto desiderata modernità parigina, questi edifici [in ferro], generalmente aperti, avevano grandi vani, strutture modulate e coperture in ferro, lanternini e lanterne veneziane in vetro, dimostrando meticolosità attraverso la flessibilità degli spazi, l'illuminazione e la ventilazione naturale ${ }^{42}$.

\footnotetext{
${ }^{41}$ Le informazioni tratte dalla descrizione del mercato nuovo provengono dal «Jornal do Brasil» di Rio de Janeiro. «O Novo Mercado, Inauguração», in Jornal do Brasil, 14 dicembre 1907, p. 3. ${ }^{42}$ JUNIOR, José Vanildo de Oliveira, Fluxograma do processo de planejamento arquitetônico aplicados a mercados públicos, Dissertação de Mestrado em Engenharia Urbana, Universidade Federal da Paraíba, 2006, pp. 40-42.
} 
Il mercato nuovo carioca pretendeva di attenersi ai criteri di ordine e igiene che si tentava di imporre alla città, oltre a rendere possibile un maggior controllo sul commercio al dettaglio. Questi nuovi criteri furono evidenti nello stile della costruzione e nell'applicazione delle nuove tecnologie al progetto, il che testimonia la buona disposizione del gruppo di impresari della Companhia Mercado Municipal ad investire, giustificata dai guadagni che aspiravano ad ottenere.

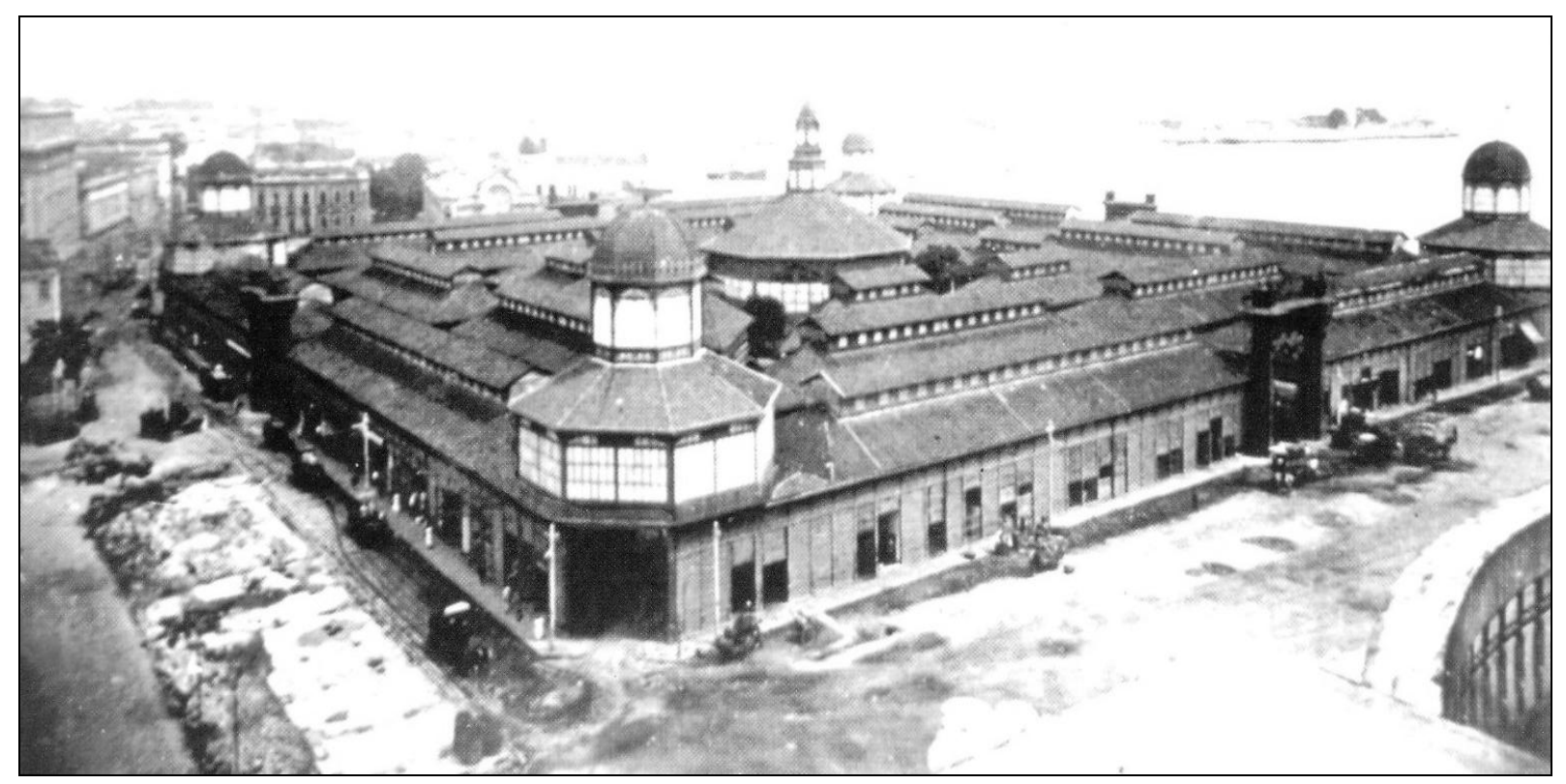

Fig. 2. Il mercato municipale di Rio de Janeiro nella fase conclusiva delle opere e dell'urbanizzazione dei dintorni. Acervo Fundação Biblioteca Nacional - Brasile.

Dal servizio del «Jornal do Brasil» traspare la preoccupazione che quello spazio si consolidasse come luogo di sociabilità e di civiltà per Rio. Infine, Rio si civilizza! L'intenzione era attrarre in quello spazio della città gli stessi uomini che indossavano cappotti di cachemire chiari, con le loro signore abbigliate con vestiti alla moda parigina. La buona società43 carioca, dopo una gradevole passeggiata nel nuovo viale, poteva approfittare della comodità e della praticità degli acquisti o, al contempo, mangiare nei ristoranti che erano lì in funzione, conversando in francese nel «patio alberato e cementato» e illuminato da «moderni lampioni e lampade a gas»44, prendere il tè (o al contrario il caffè), o deliziarsi con vini di ottima qualità e manicaretti della

43 Secondo Maria do Carmo Teixeira Rainho l'espressione era utilizzata per definire gli uomini e le donne liberi e bianchi, che si riconoscevano e si facevano riconoscere come membri del "mondo civilizzato". Ciò che caratterizza la "buona società" è il fatto di escludere gli schiavi e gli uomini liberi poveri, costituendosi in una società politica, nel contesto governativo, che è depositaria del dovere di dare un ordinamento alla società. RAINHO, Maria do Carmo T., $A$ cidade e a Moda. Brasília, Editora da Universidade de Brasília, 2000.

44 La descrizione che segue può essere rinvenuta nell'edizione del «Jornal do Commercio» di Rio de Janeiro. «O Novo Mercado», in Jornal do Commercio, 14 dicembre 1907, p. 3. 
cuisine française, laddove - nello stesso patio - tavoli e sedie rendevano possibile «alle persone che lo desideravano, fare un lunch frugale»45. La buona società annoverava anche 24 apparati sanitari e 32 orinatoi «dai sistemi più moderni»46, alcuni dei quali erano comprensibilmente destinati alle signore. Come ha ben osservato Carvalho, «Nella Rio riformata circolava il mondo belle-époque affascinato dall'Europa, imbarazzato dal Brasile ed in particolare dal Brasile povero e dal Brasile nero»47. Il sogno possibile dell'Europa si concretizzava negli edifici, come il Mercato Nuovo, che portavano la città al cosmopolitismo.

45 Ibidem.

${ }^{46}$ Ibidem.

47 CARVALHO, José Murilo de, Os bestializados: O Rio de Janeiro e a República que não foi, São Paulo, Companhia das Letras, 1987, p. 40. 


\section{* L'autore}

Vitor Leandro de Souza è specializzando del corso di Pós-Graduação in "História Social" (PPGHIS) della Universidade Federal do Rio de Janeiro (UFRJ); si è laureato in História presso l'Universidade Federal Fluminense (2011). I suoi ambiti di ricerca sono la storia sociale, la storia di Rio de Janeiro, la storia della fotografia e l'urbanistica.

URL: < http://studistorici.com/progett/autori/\#DeSouza >

\section{Per citare questo articolo:}

De SOUZA, Vitor Leandro, «Le piazze dei mercati a Rio de Janeiro (1840-1908): tra sociabilità e modernità», Diacronie. Studi di Storia Contemporanea: Sulle tracce delle idee, 29/12/2012,

URL:< http://www.studistorici.com/2012/12/29/de-souza_numero_12/ >

Diacronie Studi di Storia Contemporanea $\beta$ www.diacronie.it

Risorsa digitale indipendente a carattere storiografico. Uscita trimestrale. redazione.diacronie@hotmail.it

Comitato di redazione: Marco Abram - Jacopo Bassi - Luca Bufarale - Gianluca Canè - Alessandro Cattunar - Alice De Rensis - Barbara Galimberti - Deborah Paci - Fausto Pietrancosta - Matteo Tomasoni - Luca Zuccolo

Diritti: gli articoli di Diacronie. Studi di Storia Contemporanea sono pubblicati sotto licenza Creative Commons 2.5 Possono essere riprodotti a patto di non modificarne i contenuti e di non usarli per fini commerciali. La citazione di estratti è comunque sempre autorizzata, nei limiti previsti dalla legge. 